\title{
About the possible options for models of convective heat transfer in closed volumes with local heating source
}

\author{
Vyacheslav I. Maksimov, Tatiana A. Nagornova and Igor A. Shestakov \\ Institute of Power Engineering, National research Tomsk Polytechnic University, Tomsk 634050, \\ Russia
}

\begin{abstract}
Results of mathematical modeling of convective heat transfer in air area surrounded on all sides enclosing structures, in the presence of heat source at the lower boundary of the media are presented. Solved the system of differential equations of unsteady Navier-Stokes equations with the appropriate initial and boundary conditions. The process of convective heat transfer is calculated using the models of turbulence Prandtl and PrandtlReichard. Takes into account the processes of heat exchange region considered with the environment. Is carried out the analysis of the dimensionless heat transfer coefficient at interfaces "air - enclosures". The distributions average along the gas temperature range are obtained.
\end{abstract}

\section{Introduction}

Recently, increasing attention is paid to the study of natural convection processes [1]. The study of convective heat transfer allows the selection of optimal operating conditions in any area and appropriate technical solutions. But in the simulation of convective flows in large areas often have to take into account the turbulence [2]. One of the important issues in solving the problem of motion of air or liquid is a model of turbulence [3]. The monograph $\mathrm{H}$. Schlichting [4] refers to the validity of the application of semi-empirical formulas for the calculation of free turbulence (turbulent flow without restricting walls). [5] Therefore, the question of the possible variants of the model of convective heat transfer in confined spaces with local heating is highly relevant.

Purpose of work - analysis of two different approaches to the modeling of free-convective heat transfer in turbulent convection mode (model of Prandtl and Prandtl-Reichard).

\section{Physical model}

For the physical formulation of the problem is selected on a fairly simple geometry closed rectangular area, which includes the air (1) and enclosures (2). On three external borders are accepted of thermal insulation condition and on the left outer boundary of $X=0$ are implemented conditions of heat exchange with the environment. The entire perimeter of the interface specified boundary conditions

This is an Open Access article distributed under the terms of the Creative Commons Attribution License 4.0, which permits unrestricted use, distribution, and reproduction in any medium, provided the original work is properly cited. 


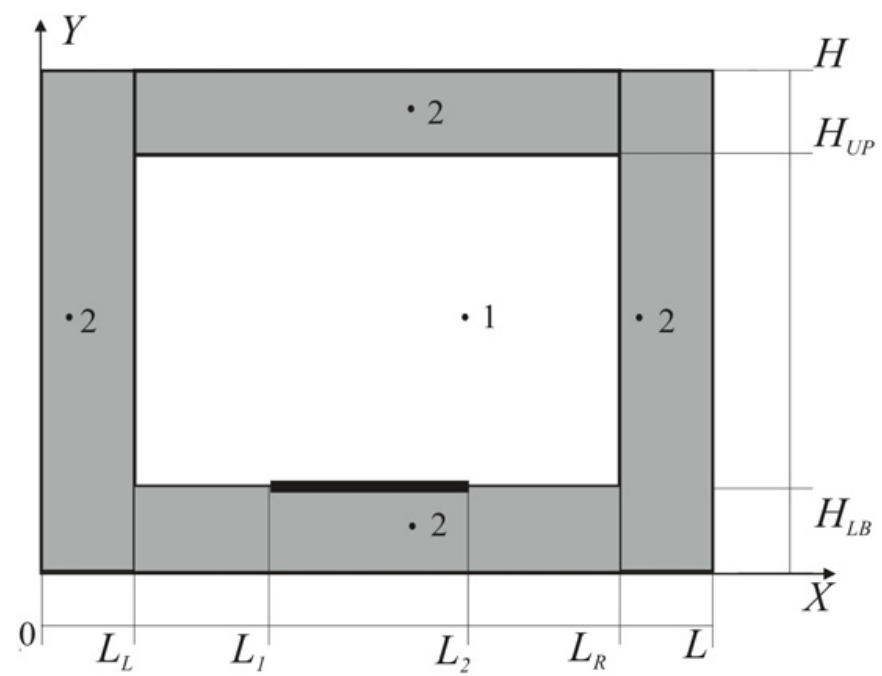

Figure 1. Area solution to the problem: 1 - air; 2 - building envelope; $L$ - width of the decision; $H$ - height field solutions; Indexes: $L, R, U P, L B$ - respectively left, right, upper and lower boundaries between media.

of the fourth kind (equality of heat flux and temperature) except for the section on the bottom border, where there is a source of heat $\left(L_{1}<X<L_{2}\right)$.

\section{Mathematical model and method of solution}

The numerical simulation of free convection in the closed domain taking into account turbulence is carried out by two methods. Problem Statement included a system of differential equations of NavierStokes equations for gas and thermal conductivity for the solid walls. Turbulent component taken into account the implementation of algebraic models of Prandtl-Reichard and Prandtl [6]. Simulation was carried out in the MatLab.

The mathematical formulation takes a similar $[7,8]$ and has the form:

$$
\begin{aligned}
& \frac{1}{\mathrm{Sh}} \frac{\partial \Omega}{\partial \tau}+U \frac{\partial \Omega}{\partial X}+V \frac{\partial \Omega}{\partial Y}=\frac{\partial^{2}}{\partial X^{2}}\left[\left(\frac{1}{\sqrt{\mathrm{Gr}}}+\frac{1}{\operatorname{Re}_{t}}\right) \Omega\right]+\frac{\partial^{2}}{\partial Y^{2}}\left[\left(\frac{1}{\sqrt{\mathrm{Gr}}}+\frac{1}{\mathrm{Re}_{t}}\right) \Omega\right]+\frac{1}{2} \frac{\partial \Theta}{\partial X}, \\
& \frac{\partial^{2} \Psi}{\partial X^{2}}+\frac{\partial^{2} \Psi}{\partial Y^{2}}=-2 \Omega \\
& \frac{1}{\mathrm{Sh}} \frac{\partial \Theta}{\partial \tau}+U \frac{\partial \Theta}{\partial X}+V \frac{\partial \Theta}{\partial Y}=\frac{\partial}{\partial X} {\left[\left(\frac{1}{\operatorname{Pr} \sqrt{\mathrm{Gr}}}+\frac{1}{\operatorname{Re}_{t}}\right) \Theta\right]+\frac{\partial}{\partial X}\left[\left(\frac{1}{\operatorname{Pr} \sqrt{\mathrm{Gr}}}+\frac{1}{\operatorname{Re}_{t}}\right) \Theta\right], } \\
& \frac{1}{\mathrm{Fo}} \frac{\partial \Theta}{\partial \tau}=\frac{\partial^{2} \Theta}{\partial X^{2}}+\frac{\partial^{2} \Theta}{\partial Y^{2}} .
\end{aligned}
$$

The initial conditions for the system of Eqs. (1-4):

$$
\Psi(X, Y, 0)=\Omega(X, Y, 0)=0, \Theta(X, Y, 0)=0 .
$$

The boundary conditions at the external borders of the field solutions:

$$
\frac{\partial \Theta(X, Y, \tau)}{\partial n}=0
$$


On the left the outer boundary:

$$
\frac{\partial \Theta(X, Y, \tau)}{\partial Y}=\mathrm{Bi} \cdot \Theta(X, Y, \tau)+\mathrm{Bi} \cdot\left(\Theta_{e}\right),
$$

At the boundaries of the solid walls and gas, the conditions:

$$
\begin{gathered}
\frac{\partial \Psi(X, Y, \tau)}{\partial n}=0, \quad \frac{\partial \Theta_{1}(X, Y, \tau)}{\partial n}=\lambda_{1,2} \frac{\partial \Theta_{2}(X, Y, \tau)}{\partial n}, \\
\Theta_{1}(X, Y, \tau)=\Theta_{2}(X, Y, \tau) . \\
\Theta(X, Y, \tau)=1, Y=\frac{H_{L B}}{L}, \quad L_{1}<X<L_{2}, \quad 0<\tau<\frac{t}{t_{0}},
\end{gathered}
$$

where $\Theta$-dimensionless temperature; $\Psi$-dimensionless stream function; $\Omega$ - dimensionless vorticity; $\tau$ - dimensionless time; $X, Y$ - dimensionless Cartesian coordinates; $U, V$ - dimensionless speed; $\mathrm{Bi}=\alpha L / \lambda-$ Biot number; $\alpha-$ coefficient of heat transfer between the external environment and the region considered decisions, $\mathrm{W} /\left(\mathrm{m}^{2} \cdot K\right) ; \lambda$ - thermal conductivity of the solid wall, $\mathrm{W} /(\mathrm{m} \cdot K) ; \lambda_{1,2}-$ relative thermal conductivity; $n$ - vector normal to the surface; $\mathrm{Gr}=g \beta L^{3}\left(T_{i t}-T_{0}\right) / \nu^{2}$ - Grashof number; $\operatorname{Pr}=v / a$ - Prandtl number; $\operatorname{Re}=2 V L / v_{t}$ - Reynolds number; $v_{t}$ - turbulent kinematic viscosity coefficient; $T$ - temperature, $K ; T_{0}$ - temperature at the initial time, $K ; T_{i t}$-temperature scale, $K ; \beta$ - thermal expansion coefficient, $K^{-1} ; g-$ acceleration of gravity, $\mathrm{m} / \mathrm{s}^{2} ;$ Fo $=a t_{0} / L^{2}-$ Fourier number; $a$ - thermal diffusivity, $\mathrm{m}^{2} / \mathrm{s} ; t$ - time, c; $t_{0}$ - time scale, $\mathrm{s}$.

Equations (1) - (4) with the appropriate initial and boundary conditions (5)-(9) are solved by finite difference method on a uniform grid. In solving the problem (1)-(9) algorithm was used [79], developed for the numerical solution of natural convection in a closed rectangular regions with local sources of energy. Turbulence in the flow of heated air is described in accordance with the basic provisions of [6], the change in viscosity with increasing air velocity. Calculation of viscosity held in the framework of the Prandtl-Reichard [6]:

$$
v_{t}=\chi\left[U_{\max }-U_{\min }\right] \delta(x),
$$

где $U_{\max }$ и $U_{\min }-$ minimum and maximum values of the velocity in the layer, $\mathrm{m} / \mathrm{c} ; \delta$ - the half-width of the mixing layer, $\mathrm{m} ; \chi$ - empirical dimensionless parameter, constant over the layer thickness, $x-$ distance measured in the direction of flow, $m$.

Prandtl model [6] corresponds to the definition of the eddy viscosity by the formula:

$$
v_{t}=l_{m}^{2}\left|\frac{\partial \bar{v}_{x}}{\partial y}\right|, \quad l_{m}=k \cdot y,
$$

где $l_{m}$ - mixing length; $x, y$ - coordinates, $\mathrm{m} ; \quad v$ - component of velocity, $\mathrm{m} / \mathrm{s} ; k$ - universal constant of proportionality is independent of the Reynolds number.

\section{Results and discussion}

The results of mathematical modeling of free-convective flows in a closed region in the presence of heat source are presented in Figs. 2-6. Numerical studies were conducted at the temperature: initial $\Theta_{0}=0$, the heat source $-\Theta i t=1$, the environment $-\Theta_{e}=0$.

As a result the numerical solution of task are obtained the temperature distributions for the region being investigated and the contour line of current function, which characterize basic laws governing the process of heat transfer (Figs. 2-3).

On Figs. 2a and 3a, it is seen that at short times, for example $(\tau=50)$, the heat sink on the left outer border still has no significant impact in the field of air is formed symmetrical temperature field. 

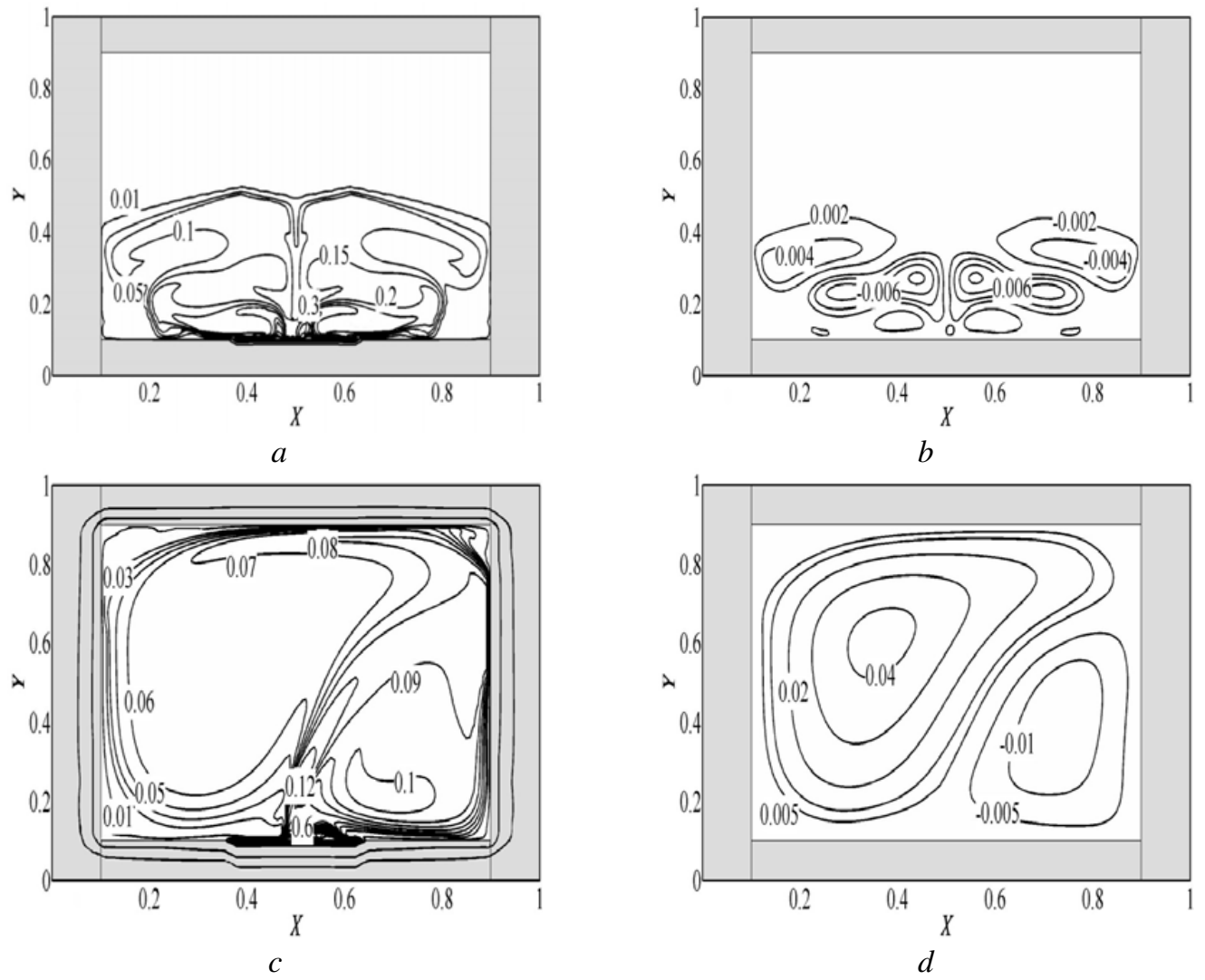

Figure 2. Temperature field $(\mathrm{a}, \mathrm{c})$ and contour lines of the stream function $(\mathrm{c}, \mathrm{d})$ for the Prandtl model with different $\tau: \tau=50(\mathrm{a}, \mathrm{b}), \tau=500(\mathrm{c}, \mathrm{d})$.

The temperature distribution (Figs. 2 and 3) clearly illustrates the effect of lift, which is due to the inhomogeneity of the temperature field. Analysis of the medium-gas temperatures allows to speak about their significantly uneven distribution in time due to the influence of buoyancy force (Fig. 4).

With increasing time parameter becomes visible vortex formation. The main flow of the heated air is displaced to the right edge of the media, the top of which there is an intensive heat exchange between the hot gas and cold walling. The consequence of this is a (Fig. 5) an increase in the average Nusselt number at the boundary $X=L_{R}$.

Temperature fields obtained with the implementation of two different approaches to the modeling of turbulence sufficiently comparable, the direction of movement of the heated air coincide.

Figure 5-6 shows the variation of the average Nusselt number on the vertical $\left(X=L_{L}, X=L_{R}\right)$ interface between the media in the time determined by the expression [9]:

$$
N u_{a v y}=\frac{1}{H_{U P}-H_{L B}} \int_{H_{L B}}^{H_{U P}}\left|\frac{\partial \Theta}{\partial X}\right|_{X=L_{L}} d Y ; \quad N u_{a v y}=\frac{1}{H_{U P}-H_{L B}} \int_{H_{L B}}^{H_{U P}}\left|\frac{\partial \Theta}{\partial X}\right|_{X=L_{R}} d Y .
$$

It may be noted that these results illustrate the stabilization modes of heat transfer at interfaces "air solid wall" at relatively large time $(\tau=500)$, except the right of the interface "air - enclosures". In this case the average Nusselt number with time grow with increasing $\tau$ more than 2 times. The results of 
Thermophysical Basis of Energy Technologies

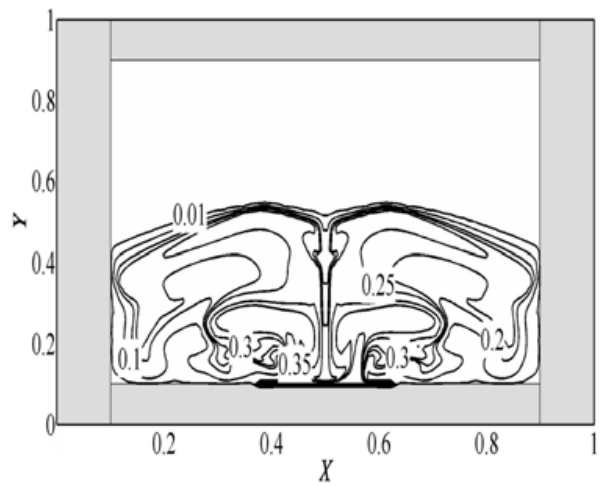

$a$

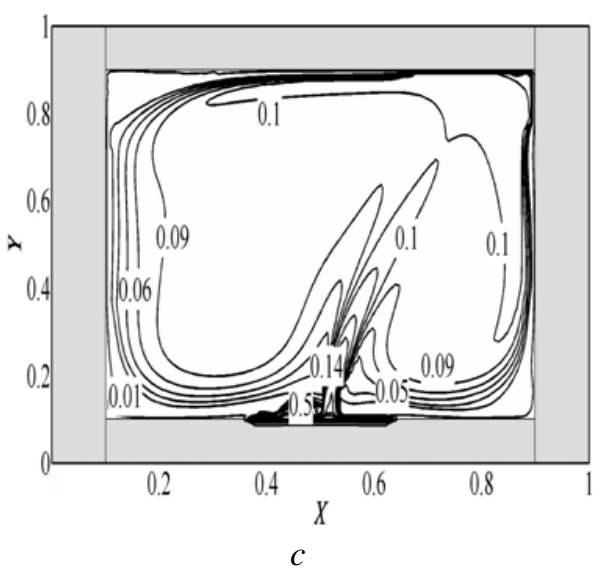

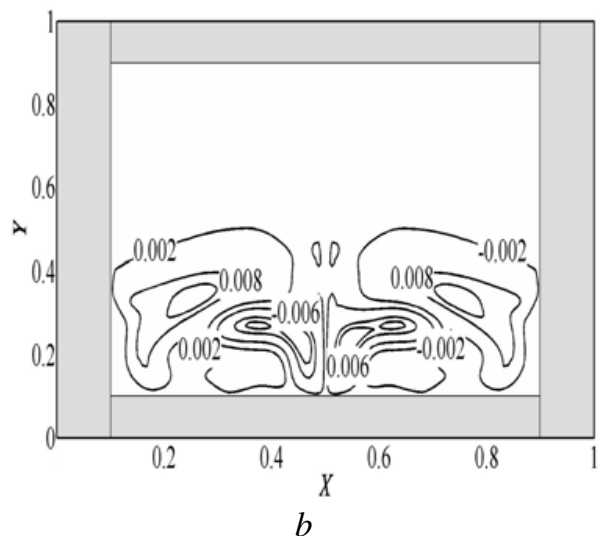

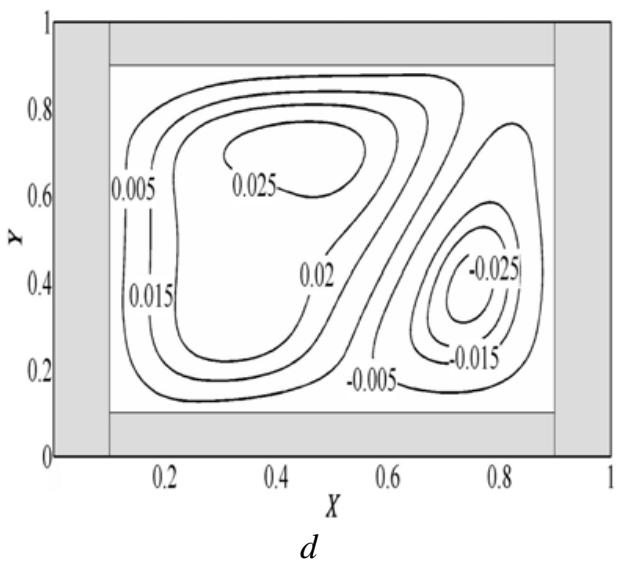

Figure 3. Temperature field (a, c) and contour lines of the stream function (c, d) for the Prandtl model with different $\tau: \tau=50(\mathrm{a}, \mathrm{b}), \tau=500(\mathrm{c}, \mathrm{d})$.

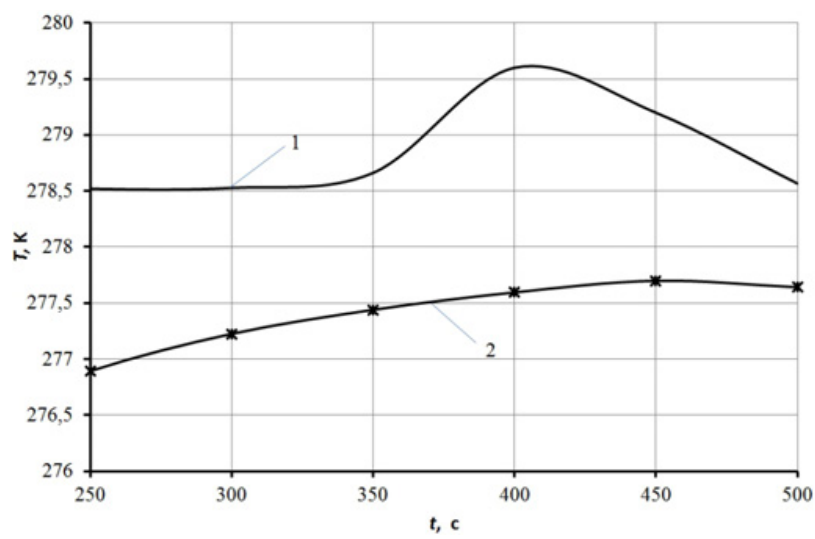

Figure 4. Dependence of the average air temperature on the time: 1 - model of Prandtl-Reichard, 2 - Prandtl model. 


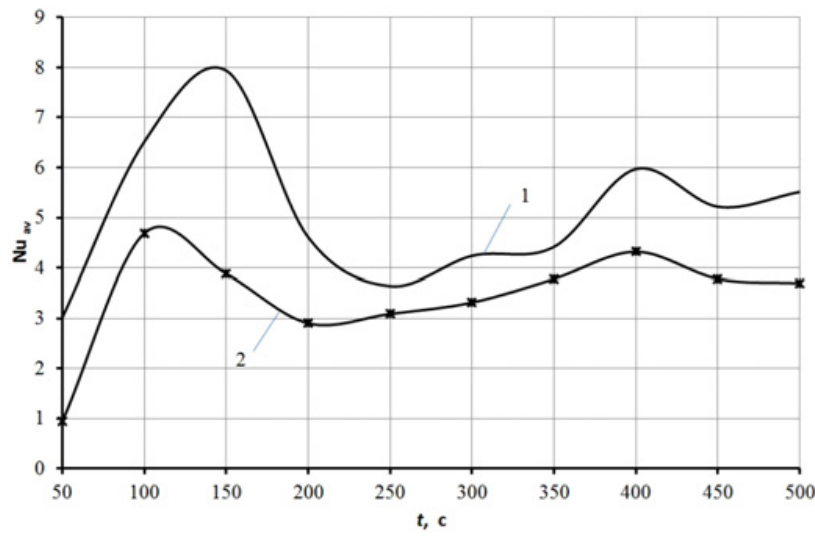

Figure 5. Dependence of the average Nusselt number on the time for the boundary $X=L_{L}: 1-$ model of PrandtlReichard, 2 - Prandtl model.

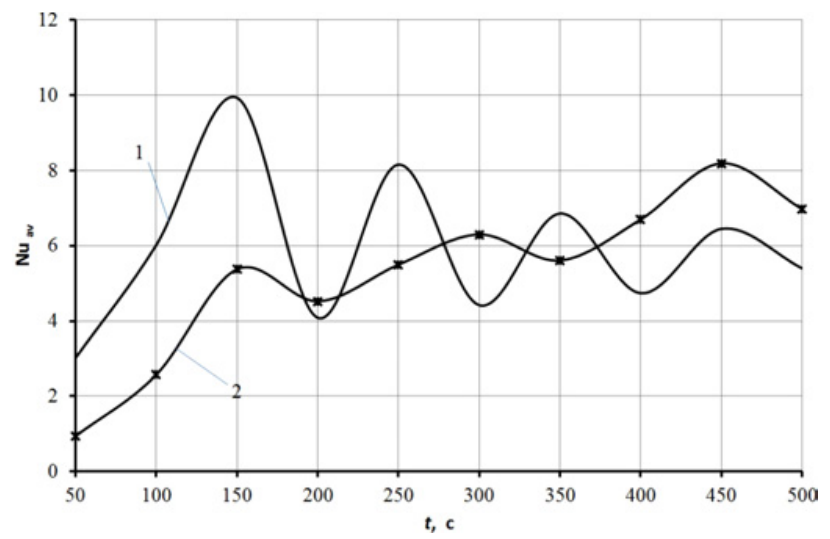

Figure 6. Dependence of the average Nusselt number on the time for the boundary $X=L_{R}: 1-$ model of PrandtlReichard, 2 - Prandtl model.

numerical simulations show the possibility of using different approaches to the description of thermal conditions of turbulent convection in confined areas with local sources of heat.

\section{Conclusion}

Was carried out a theoretical study of turbulent natural convection in a system with a heat source. The results allow to evaluate the nature of the flow and heat transfer intensity of the analyzed process, as well as new information that not only characterizes the convective flow regime, but is optional for the construction and testing of complex turbulence models, taking into account the peculiarities of convective heat transfer in large confined spaces with local heating. The results of mathematical modeling allow to draw conclusions on the applicability of the algebraic model of Prandtl for the consideration turbulent flows for natural convection in a geometrically simple domains [10]. Use of this model is advisable in the case when the movement of the heated air is the source of heat due to the lifting force [5]. It should be noted that the use of the algebraic model for calculating the free convective flow 
in the turbulent regime is much simpler and requires less computation time than the more sophisticated models of turbulence.

Work performed under the research state assignment "Science" (Code of Federal Target Scientific and Technical Program 2.1321.2014).

\section{References}

[1] S.V. Solov'ev, L.S. Haenko, Modelling of natural convection in an inclined cavity, Herald of Pacific National University: physical and mathematical sciences, 16, 1, (2010) 27-34

[2] G.V. Tkachenko, B.A. Uryukov, Model of turbulent natural convection near a vertical wall on the basis of the hypothesis S.S. Kutateladze, IV Russian National Conference on Heat Transfer, 3 (2006) 160-163

[3] V.T. Movchan, E.A. Shkvarov, Mathematical modeling of boundary layers, Applied Hydromechanics, 79, 7 (2005) P. 73-85

[4] G. Shlihning, Boundary layer theory, Moscow: Nauka, (1974) 712 p.

[5] W. Schmidt, Turbulente Ausbreitung eines Stormes erhitzter Luft, ZAMM, 21, 5/6 (1941)

[6] I.A. Belov, S.A. Isaev, Modeling of turbulent flows: Study Guide. Baltic State Technical University.- St.P., (2001) 108 p.

[7] G.V. Kuznetsov, N.I. Kurilenko, V.I. Maksimov, G.Ya. Mamontov, T.A. Nagornova, Journal of Engineering Physics and Thermophysics. 86 (2013) 519-524

[8] V. I. Maksimov, T.A. Nagornova, EPJ Web of Conferences 76 (2014), Article number 01006

[9] G.V. Kuznetsov, M.A. Sheremet, Conguate natural convection in enclosure with local heat sources, Computational thermal sciences. 1, 3 (2009) 341-360

[10] Y.V. Lapin, Sagittarius MH Internal flow of gas mixtures, Moscow: Nauka, (1989) 370 p. 